\title{
On Flag Vectors, the Dowling Lattice, and Braid Arrangements
}

\author{
R. Ehrenborg and M. A. Readdy \\ Department of Mathematics, Cornell University, \\ White Hall, Ithaca, NY 14853-7901, USA \\ \{jrge,readdy\}@math.cornell.edu
}

\begin{abstract}
We study complex hyperplane arrangements whose intersection lattices, known as the Dowling lattices, are a natural generalization of the partition lattice. We give a combinatorial description of the Dowling lattice via enriched partitions to obtain an explicit $E L$-labeling and then find a recursion for the flag $h$-vector in terms of weighted derivations. When the hyperplane arrangements are real they correspond to the braid arrangements $A_{n}$ and $B_{n}$. By applying a result due to Billera and the authors, we obtain a recursive formula for the cd-index of the lattice of regions of the braid arrangements $A_{n}$ and $B_{n}$.
\end{abstract}

\section{Introduction}

The cd-index is a noncommutative polynomial which gives an efficient encoding of the flag $f$-vector, equivalently the flag $h$-vector, of an Eulerian poset. The generalized Dehn-Sommerville equations [2] describe all of the linear relations that hold among the entries of the flag $f$-vector, while the cd-index removes the linear redundancies. The cd-index has been a very successful tool to answer questions about convex polytopes, including showing the flag $f$-vectors of zonotopes satisfy precisely the same affine relations as the flag $f$-vectors of all polytopes and settling the zonotopal analogue of a conjecture of Stanley, that among all zonotopes the cubical lattice has the smallest cd-index coefficientwise; see [6]. It is believed that the cd-index will be a useful invariant in determining linear inequalities in the flag $f$-vector of convex polytopes, and, more generally, Gorenstein* lattices. For the known inequalities in dimension 4, see [1, Theorem 3.10], [4], and [19].

Given its usefulness, one would naturally like to be able to compute the cd-index. The first recursion formulas for the cd-index were given by Purtill [21] for the Boolean algebra and the cubical lattice, that is, the face lattice of the $n$-simplex and the $n$-cube. In [16] the authors gave shorter recursions using derivations, as well as determined how the cd-index changes under the pyramid and prism operations. The cd-index is also 
understood for simplicial polytopes, and, more generally, Eulerian simplicial posets. Stanley [25] expressed the cd-index of a simplicial polytope in terms of its $h$-vector and certain cd-polynomials $\breve{\Phi}_{i}^{n}$ and conjectured a combinatorial interpretation for the $\check{\Phi}_{i}^{n}$. This conjecture was proved by Hetyei [17], whereas a short recursion for these polynomials in terms of a derivation was found in [16]. Cubical polytopes, more generally Eulerian cubical posets, have been studied in [14].

In this paper we study the cd-index of the braid arrangements $A_{n}$ and $B_{n}$. In order to do this we consider a more general hyperplane arrangement in complex space. Let $k$ be a positive integer and let $\zeta$ be a primitive $k$ th root of unity. Let $\mathcal{H}_{n, k}$ be the following hyperplane arrangement in $n$-dimensional complex space:

$$
\begin{array}{ll}
z_{i}=\zeta^{h} \cdot z_{j} & \text { for } \quad 1 \leq i<j \leq n \\
z_{i}=0 & \text { for } \quad 1 \leq i \leq n .
\end{array} \quad \text { and } \quad 0 \leq h \leq k-1,
$$

This hyperplane arrangement has been studied earlier in [18] and [20, Section 6.4]. The braid arrangements $A_{n}$ and $B_{n}$ correspond to the cases $k=1$ and $k=2$. The intersection lattice $L_{n, k}$ of the arrangement $\mathcal{H}_{n, k}$ is called the Dowling lattice and is a natural generalization of the partition lattice [11], [12].

We give a combinatorial description of the Dowling lattice via enriched partitions. Since the Dowling lattice is a geometric lattice, it has many $E L$-labelings. Using enriched partitions, we obtain an explicit EL-labeling and determine the set of lists of labels of maximal chains. By understanding the structure of these lists of labels, we find a recursion for the flag $h$-vector of the Dowling lattice in terms of weighted derivations. As a corollary, the characteristic polynomial and Möbius function are determined. This, together with the $E L$-labeling, yields topological information about the order complex of this family of lattices.

When the parameter $k$ is equal to one or two, the complex hyperplane arrangement $\mathcal{H}_{n, k}$ is a hyperplane arrangement in real space. A real hyperplane arrangement has two lattices associated with it, namely the intersection lattice and the lattice of regions. In [7] the authors, together with Billera, completely determine how to compute the cd-index of the lattice of regions in terms of the intersection lattice. By applying this result to the Dowling lattice recursion, we obtain a recursive formula for the cd-index of the lattice of regions of the braid arrangements $A_{n}$ and $B_{n}$.

\section{Definitions}

All the posets we consider will be graded of rank greater than or equal to one, that is, posets $P$ having a minimal element $\hat{0}$ and a maximal element $\hat{1}$ so that $\hat{0} \neq \hat{1}$. The associated rank function will be denoted by $\rho$ and satisfy $\rho(\hat{0})=0$. For $x \leq y$ define $\rho(x, y)$ to be equal to $\rho(y)-\rho(x)$ and define the interval from $x$ to $y$ to be set $\{z: x \leq z \leq y\}$, denoted $[x, y]$. Observe that $[x, y]$ is a graded poset of rank $\rho(x, y)$.

A poset $L$ is a lattice if every two elements $x$ and $y$ has a unique greatest lower bound or meet, denoted by $x \wedge y$, and a unique least upper bound or join, denoted by $x \vee y$. A ranked lattice $L$ is semimodular if it satisfies the inequality $\rho(x)+\rho(y) \geq \rho(x \wedge y)+\rho(x \vee y)$, for all $x, y \in L$, and atomic if all of its elements can be written as a join of atoms. A lattice which is both semimodular and atomic is a geometric lattice. 
Let $P$ be a graded poset of rank $n+1$. For $S$ a subset of $\{1, \ldots, n\}$, let $P_{S}$ be the subposet of $P$ defined as $P_{S}=\{x \in P \quad: \rho(x) \in S, x=\hat{0}$, or $x=\hat{1}\}$. Let $\alpha(S)$ be the number of maximal chains in $P_{S}$, that is, $\alpha(S)$ is the number of chains in $P$ whose ranks correspond to the set $S$. Define $\beta(S)$ by the equation

$$
\beta(S)=\sum_{T \subseteq S}(-1)^{|S-T|} \cdot \alpha(T) .
$$

The $2^{n}$ entries of the flag $f$-vector and flag h-vector correspond to the values of $\alpha(S)$ and $\beta(S)$.

Let $\mathbf{a}$ and $\mathbf{b}$ be two noncommuting variables. For a subset $S$ of $\{1, \ldots, n\}$, define $u_{S}$ to be the ab-monomial $u_{1} \cdots u_{n}$ where $u_{i}=\mathbf{a}$ if $i \notin S$ and $u_{i}=\mathbf{b}$ if $i \in S$. The ab-index of a poset $P$ of rank $n+1, \Psi(P)$, is defined by

$$
\Psi(P)=\sum_{S} \beta(S) \cdot u_{S}
$$

where the sum ranges over all subsets $S$ of $\{1, \ldots, n\}$. Observe the ab-index encodes exactly the same information as the flag $h$-vector. Moreover, $\Psi(P)$ is a homogeneous polynomial of degree $n$.

The Möbius function $\mu(x, y)$ is defined for $x, y \in P$ by $\mu(x, x)=1$ and for $x<y$ in $P$ by $\sum_{x \leq z \leq y} \mu(x, z)=0$. We denote $\mu(\hat{0}, \hat{1})$ by $\mu(P)$. Then we have $\beta(S)=(-1)^{|S|+1}$. $\mu\left(P_{S}\right)$. A poset $P$ is called Eulerian if the Möbius function satisfies $\mu(x, y)=(-1)^{\rho(x, y)}$. Fine [3] observed that when $P$ is Eulerian the ab-index of $P$ can be written in terms of the noncommuting variables $\mathbf{c}=\mathbf{a}+\mathbf{b}$ and $\mathbf{d}=\mathbf{a} \cdot \mathbf{b}+\mathbf{b} \cdot \mathbf{a}$. The resulting polynomial is called the cd-index. An elementary proof of this fact appears in [25]. In the case when $P$ is the lattice of regions of a hyperplane arrangement (or more generally, of an oriented matroid $\mathcal{M}$ ), the ab-index of $P$ can be written as a polynomial with integer coefficients in the noncommuting variables $\mathbf{c}$ and $2 \cdot \mathbf{d}$. The resulting polynomial is called the c-2d-index; see [6].

Let $\mathbb{Z}\langle\mathbf{a}, \mathbf{b}\rangle$ be the ring of polynomials in the variables $\mathbf{a}$ and $\mathbf{b}$, and let the degree of $\mathbf{a}$ and $\mathbf{b}$ be 1 . Let $\mathbb{Z}\langle\mathbf{c}, 2 \mathbf{d}\rangle$ denote the subring of $\mathbb{Z}\langle\mathbf{a}, \mathbf{b}\rangle$ spanned by the elements $\mathbf{c}=\mathbf{a}+\mathbf{b}$ and $2 \mathbf{d}=2 \mathbf{a b}+2 \mathbf{b a}$. Thus $\mathbf{c}$ has degree 1 and $2 \mathbf{d}$ has degree 2 . For a poset $P$, let $P^{*}$ denote the dual poset. The poset $P^{*}$ has the same underlying set as $P$ but with the order relation $x \leq_{P^{*}} y$ if $x \geq_{P} y$. Similarly, for an ab-monomial $v=v_{1} v_{2} \cdots v_{n}$, let $v^{*}=v_{n} \cdots v_{2} v_{1}$. By linearity we extend this operation to be an involution on $\mathbb{Z}\langle\mathbf{a}, \mathbf{b}\rangle$. Since $\mathbf{c}^{*}=\mathbf{c}$ and $2 \mathbf{d}^{*}=2 \mathbf{d}$, the involution restricts to $\mathbb{Z}\langle\mathbf{c}, 2 \mathbf{d}\rangle$ by reading the $\mathbf{c}-2 \mathbf{d}$-monomials backwards. Observe for a graded poset $P$ we have $\Psi\left(P^{*}\right)=\Psi(P)^{*}$.

\section{Techniques for Computing the ab- and cd-Indexes}

When a poset $P$ has an $R$-labeling, there is a known method to compute the ab-index of $P$. This method will be extended so that one can compute the cd-index of the lattice of regions of hyperplane arrangements.

Recall an edge-labeling $\lambda$ of a locally finite poset $P$ is a map which assigns to each edge in the Hasse diagram of $P$ an element from some poset $\Lambda$. For us $\Lambda$ will always 
be a linearly ordered set. If $y$ covers $x$ in $P$, then we denote the label on the edge $(x, y)$ by $\lambda(x, y)$. A maximal chain $x=x_{0} \prec x_{1} \prec \cdots \prec x_{k}=y$ in an interval $[x, y]$ in $P$ is called rising if the labels are weakly increasing with respect to the order of the poset $\Lambda$, that is, $\lambda\left(x_{0}, x_{1}\right) \leq_{\Lambda} \lambda\left(x_{1}, x_{2}\right) \leq_{\Lambda} \cdots \leq_{\Lambda} \lambda\left(x_{k-1}, x_{k}\right)$. An edge-labeling is called an $R$-labeling if for every interval $[x, y]$ in $P$ there is a unique rising maximal chain in $[x, y]$.

Let $P$ be a poset of rank $n+1$ with $R$-labeling $\lambda$. For a maximal chain $c=\{\hat{0}=$ $\left.x_{0} \prec x_{1} \prec \cdots \prec x_{n+1}=\hat{1}\right\}$, the descent set $D(c)$ is the set $D(c)=\left\{i: \lambda\left(x_{i-1}, x_{i}\right)>_{\Lambda}\right.$ $\left.\lambda\left(x_{i}, x_{i+1}\right)\right\}$. Observe that $D(c)$ is a subset of the set $\{1, \ldots, n\}$. Our interest in $R$ labelings stems from the following result of Björner and Stanley (see Theorem 2.7 of [9]):

Proposition 3.1. Let $P$ be a graded poset that admits an R-labeling. Then $\beta(S)$ is equal to the number of maximal chains $c$ with descent set $S$.

From this result we obtain the following corollary, which was observed in [15].

Corollary 3.2. Let $P$ be a graded poset of rank $n+1$. Let $\lambda$ be an R-labeling of $P$. Then the ab-index of $P$ is given by

$$
\Psi(P)=\sum_{c} u_{D(c)},
$$

where the sum is over all maximal chains $c$ of the poset $P$.

An EL-labeling of a graded poset $P$ is an $R$-labeling such that in each interval $[x, y]$ the unique rising chain is lexicographically least among all chains in the interval $[x, y]$. If a poset possesses an $E L$-labeling, then it is known that the chain complex of the poset $P$ is shellable [9]. Moreover, the chain complex is homotopy equivalent to a wedge of spheres.

An $E L$-labeling of a geometric lattice $L$ can be obtained as follows; see Example 3.13.5 of [24]. Let $\Lambda$ denote the set of atoms of $L$ and let there be a total ordering on the atoms. The label on the edge $x \prec y$ may be described by

$$
\lambda(x, y)=\min \{a \in \Lambda: x \vee a=y\} .
$$

Observe that with this $E L$-labeling two different chains will have two different lists of labels.

We now turn our attention to hyperplane arrangements. Let $\mathcal{H}$ be a hyperplane arrangement in $\mathbb{R}^{n}$. We assume that $\mathcal{H}=\left\{H_{e}: e \in E\right\}$ is essential, that is, $\bigcap_{e \in E} H_{e}=\{0\}$. Associated with a hyperplane arrangement are two lattices. The intersection lattice of $\mathcal{H}$ is the lattice on the set of subspaces $\left\{\bigcap_{e \in S} H_{e}: S \subseteq E\right\}$ ordered by reverse inclusion. Thus $\mathbb{R}^{n}$ is the minimal element, $\{0\}$ is the maximal element, and the hyperplanes in the arrangement are the atoms. Each hyperplane $H$ in $\mathcal{H}$ cuts $\mathbb{R}^{n}$ into three pieces, namely, the hyperplane itself and two open half-spaces. Together all the hyperplanes in $\mathcal{H}$ cut $\mathbb{R}^{n}$ into relative open cones, which we call regions. Let $R$ be the set of regions. The set $R$ forms a poset by the order relation $C \leq C^{\prime}$ if the closure of the region $C$ is contained in 
the topological closure of the region $C^{\prime}$. We adjoin a maximal element to $R$ to obtain a lattice, called the lattice of regions of the hyperplane arrangement $\mathcal{H}$.

Bayer and Sturmfels [5, Theorem 3.4] showed that the flag $f$-vector of the lattice of regions $R$ depends only on the intersection lattice $L$. This dependency was showed in an explicit manner in [7].

The lattice of regions is an Eulerian poset, hence it has a cd-index. In fact it has a c-2d-index, that is, the cd-index may be written as a polynomial in $\mathbf{c}$ and $2 \mathbf{d}$ with integer coefficients. Theorem 3.4 shows how to compute the $\mathbf{c}-2 \mathbf{d}$-index of the lattice of regions.

Definition 3.3. Define a linear function $\omega: \mathbb{Z}\langle\mathbf{a}, \mathbf{b}\rangle \rightarrow \mathbb{Z}\langle\mathbf{c}, 2 \mathbf{d}\rangle$ as follows: For an abmonomial $v$ compute $\omega(v)$ by replacing each occurrence of ab in the monomial $v$ with $2 \mathbf{d}$, then replacing the remaining letters with c's. Extend this definition by linearity to ab-polynomials.

Theorem 3.4 [7]. Let $\mathcal{H}$ be a hyperplane arrangement, let $R$ be the lattice of regions of $\mathcal{H}$, and let $L$ be the intersection lattice of $\mathcal{H}$. Then the $\mathbf{c}-2 \mathbf{d}$-index of $R$ is given by

$$
\Psi(R)=\omega(\mathbf{a} \cdot \Psi(L))^{*}
$$

For instance, the intersection lattice of the braid arrangement $A_{3}$ has the ab-index $\mathbf{a a}+5 \cdot \mathbf{b a}+6 \cdot \mathbf{a b}+6 \cdot \mathbf{b b}$. Hence the $\mathbf{c}-2 \mathbf{d}$-index of the lattice of regions of the arrangement $A_{3}$ is given by

$$
\begin{aligned}
\omega(\mathbf{a a a}+5 \cdot \mathbf{a b a}+6 \cdot \mathbf{a a b}+6 \cdot \mathbf{a b b})^{*} & =\left(\mathbf{c}^{3}+11 \cdot 2 \mathbf{d} \cdot \mathbf{c}+6 \cdot \mathbf{c} \cdot 2 \mathbf{d}\right)^{*} \\
& =\mathbf{c}^{3}+11 \cdot \mathbf{c} \cdot 2 \mathbf{d}+6 \cdot 2 \mathbf{d} \cdot \mathbf{c}
\end{aligned}
$$

By combining Stanley's EL-labeling of geometric lattices with Corollary 3.2 and Theorem 3.4, we have the following corollary.

Corollary 3.5. The c-2d-index of the lattice of regions $R$ is given by

$$
\Psi(R)=\sum_{c} \omega\left(\mathbf{a} \cdot u_{D(c)}\right)^{*}
$$

where the sum ranges over all maximal chains $c$ in the intersection lattice $L$.

\section{The Dowling Lattice}

The Dowling lattice $L_{n, k}$ is the intersection lattice of the complex hyperplane arrangement $\mathcal{H}_{n, k}$. Since it is an intersection lattice, it follows that the Dowling lattice is a geometric lattice of rank $n$. Observe $L_{n, 1}$ is isomorphic to $\Pi_{n+1}$, the partition lattice of rank $n$. Each of the hyperplanes in $\mathcal{H}_{n, k}$ is an atom in the Dowling lattice $L_{n, k}$, hence $L_{n, k}$ has $k \cdot\left(\begin{array}{l}n \\ 2\end{array}\right)+n$ atoms.

The Dowling lattice $L_{n, k}$ has the following combinatorial description. Define an enriched block $\widetilde{B}=(B, f)$ to be a nonempty subset $B$ of $\{1, \ldots, n\}$ and a function 
$f: B \rightarrow \mathbb{Z}_{k}$. We say two enriched blocks $\widetilde{B}=(B, f)$ and $\widetilde{C}=(C, g)$ are equivalent if $B=C$ and the functions $f$ and $g$ differ only by a constant. Hence there are only $k^{|B|-1}$ possible ways to enrich the nonempty set $B$, up to equivalence. When $k=1$ there is exactly one way to enrich a block, that is, there is no enrichment. Let $\widetilde{B}$ and $\widetilde{C}$ be two disjoint enriched blocks and let $i$ be an element in $\mathbb{Z}_{k}$. We can define a function $h$ on the block $B \cup C$ by

$$
h(b)=\left\{\begin{array}{lll}
f(b) & \text { if } \quad b \in B, \\
g(b)+i & \text { if } \quad b \in C .
\end{array}\right.
$$

Since $i$ can be chosen in $k$ possible ways, there are $k$ possible ways to merge two enriched blocks.

For $E$ a subset of $\{1, \ldots, n\}$, an enriched partition $\widetilde{\pi}=\left\{\widetilde{B}_{1}, \ldots, \widetilde{B}_{m}\right\}$ on the set $E$ is a partition $\pi=\left\{B_{1}, \ldots, B_{m}\right\}$ of $E$, where each block $B_{i}$ is enriched with a function $f_{i}$. Observe that on the empty set there is exactly one enriched partition, namely the empty partition.

Define the lattice $L_{n, k}^{\prime}$ to be the set

$L_{n, k}^{\prime}=\{(\tilde{\pi}, Z): Z \subseteq\{1, \ldots, n\}$ and $\tilde{\pi}$ is an enriched partition of $\bar{Z}=\{1, \ldots, n\}-Z\}$.

We call the set $Z$ the zero set. Define the order relation on $L_{n, k}^{\prime}$ by the following two relations: $\left(\left\{\widetilde{B}_{1}, \widetilde{B}_{2}, \ldots, \widetilde{B}_{m}\right\}, Z\right)<\left(\left\{\widetilde{B}_{2}, \ldots, \widetilde{B}_{m}\right\}, Z \cup B_{1}\right)$ and $\left(\left\{\widetilde{B}_{1}, \widetilde{B}_{2}, \ldots, \widetilde{B}_{m}\right\}, Z\right)<$ $\left(\left\{\widetilde{B}_{1} \cup \widetilde{B}_{2}, \ldots, \widetilde{B}_{m}\right\}, Z\right)$. The first relation says that a block is allowed to merge with the zero set. The second relation says that two blocks are allowed to be merged together.

Given $(\tilde{\pi}, Z) \in L_{n, k}^{\prime}$, construct the corresponding subspace by

$$
\begin{aligned}
\zeta^{f(i)} \cdot z_{i} & =\zeta^{f(j)} \cdot z_{j} & & \text { for } \quad i, j \in B \\
z_{i} & =0 & & \text { for } \quad i \in Z .
\end{aligned} \quad \text { and } \quad \widetilde{B}=(B, f) \in \tilde{\pi},
$$

It is straightforward to see that this is an isomorphism between $L_{n, k}$ and $L_{n, k}^{\prime}$.

Proposition 4.1. The two lattices $L_{n, k}$ and $L_{n, k}^{\prime}$ are isomorphic.

By the compositional exponential formula, see for instance, Chapter 5 of [27], we obtain the next lemma.

Lemma 4.2. Let $a_{n}$ be the number of elements in the Dowling lattice $L_{n, k}$. Then the exponential generating function for the sequence $a_{n}$ is given by

$$
\sum_{n \geq 0} a_{n} \cdot \frac{x^{n}}{n !}=\exp (x) \cdot \exp \left(\frac{1}{k} \cdot(\exp (k \cdot x)-1)\right) \text {. }
$$

Since $L_{n, k}$ is a geometric lattice, an $R$-labeling of $L_{n, k}$ is found by giving a linear order $\Lambda$ to the atoms of $L_{n, k}$. First, denote the atom corresponding the hyperplane $z_{i}=0$ by $i$. Similarly, describe the atom corresponding to the hyperplane $z_{i}=\zeta^{h} \cdot z_{j}$ by the 
triple $(i, j, h)$, where $1 \leq i<j \leq n$ and $h \in \mathbb{Z}_{k}$. On the set of atoms of $L_{n, k}$ consider the following linear order $\Lambda$ :

- $j<_{\Lambda} j^{\prime}$ if $1 \leq j<j^{\prime} \leq n$,

- $j<_{\Lambda}\left(i^{\prime}, j^{\prime}, h^{\prime}\right)$ if $1 \leq j \leq j^{\prime} \leq n$,

- $(i, j, h)<_{\Lambda} j^{\prime}$ if $2 \leq j<j^{\prime} \leq n$,

- $(i, j, h)<_{\Lambda}\left(i^{\prime}, j^{\prime}, h^{\prime}\right)$ if one of the following three conditions holds:

- $2 \leq j<j^{\prime} \leq n$,

- $j=j^{\prime}$ and $i<i^{\prime}$, or

- $j=j^{\prime}, i=i^{\prime}$, and $h<h^{\prime}$.

Let $M_{n, k}$ be the set of lists of labels of maximal chains in $L_{n, k}$, that is,

$M_{n, k}=\left\{\left(\lambda\left(x_{0}, x_{1}\right), \ldots, \lambda\left(x_{n-1}, x_{n}\right)\right):\left(x_{0}, x_{1}, \ldots, x_{n}\right)\right.$ is a maximal chain in $\left.L_{n, k}\right\}$.

To characterize the set $M_{n, k}$, we need the following two notions. For an atom $a$ of $L_{n, k}$ we define its support $\sigma$ by

$$
\sigma(a)= \begin{cases}\{i, j\} & \text { if } \quad a=(i, j, h) \\ \{i\} & \text { if } \quad a=i .\end{cases}
$$

For an element $x$ in $L_{n, k}$ define $M(x)$ to be set

$$
M(x)=\left\{\min \left(\widetilde{B}_{i}\right): 1 \leq i \leq m\right\},
$$

where $x$ as an element in $L_{n, k}^{\prime}$ is the element $(\tilde{\pi}, Z)$ with $\widetilde{\pi}=\left\{\widetilde{B}_{1}, \ldots, \widetilde{B}_{m}\right\}$ and $\min \left(\widetilde{B}_{i}\right)$ denotes the smallest element contained in the block $B_{i}$. Observe that if $x \leq y$, then $M(y) \subseteq M(x)$. Moreover, $n-|M(x)|$ is the rank of the element $x$.

Lemma 4.3. Let $x \prec y$ be a cover relation in the Dowling lattice $L_{n, k}$. If the element $y$ is formed by merging two blocks $\widetilde{B}_{1}$ and $\widetilde{B}_{2}$ of $x$, then the label $\lambda(x, y)$ is of the form $(i, j, h)$ where $\{i, j\}=\left\{\min \left(\widetilde{B}_{1}\right), \min \left(\widetilde{B}_{2}\right)\right\}$. If the element $y$ is formed by joining a block $\widetilde{B}_{1}$ to the zero set, then the label $\lambda(x, y)$ is of the form $i$ where $i=\min \left(\widetilde{B}_{1}\right)$.

Proof. We prove the lemma in the first case. The second case follows by a similar argument. Henceforth assume the element $y$ is obtained by merging the two blocks $\widetilde{B}_{1}$ and $\widetilde{B}_{2}$ of $x$. Let $i$ be the smallest element in the block $\widetilde{B}_{1}$ and let $j$ be the smallest element in the block $\widetilde{B}_{2}$. We may assume that $i<j$. Let $h$ be the unique element in $\mathbb{Z}_{k}$ such that joining the atom $(i, j, h)$ with the element $x$ gives the element $y$. We claim that the label $\lambda(x, y)$ is given by $(i, j, h)$.

Assume that $a$ is an atom such that $a \vee x=y$. Then the atom $a$ has the form $\left(i^{\prime}, j^{\prime}, h^{\prime}\right)$, where $i^{\prime}<j^{\prime}$. Moreover, either $i^{\prime} \in \widetilde{B}_{1}$ and $j^{\prime} \in \widetilde{B}_{2}$ or $i^{\prime} \in \widetilde{B}_{2}$ and $j^{\prime} \in \widetilde{B}_{1}$. Using the fact that $i$ and $j$ are the smallest elements in their respective blocks, we obtain in the first case that $(i, j, h)<_{\Lambda} a$ or $(i, j, h)=a$. In the second case we have $i<j \leq i^{\prime}<j^{\prime}$, so we get $(i, j, h)<_{\Lambda} a$.

Corollary 4.4. Let $x \prec y$ be a cover relation in the Dowling lattice $L_{n, k}$. Then $\sigma(\lambda(x, y)) \subseteq M(x)$. 
Proof. The label $\lambda(x, y)$ is either equal to $(i, j, h)$ or $i$. Consider the first case. Then $\sigma(\lambda(x, y))=\{i, j\}$. By Lemma 4.3, the elements $i$ and $j$ are the smallest elements from their respective blocks. Hence $\{i, j\} \subseteq M(x)$. Similarly, in the second case we obtain $\sigma(\lambda(x, y))=\{i\} \subseteq M(x)$.

Lemma 4.5. Let $x \prec y$ be a cover relation in the Dowling lattice $L_{n, k}$ and assume that $y \leq z$. Then the element $\max (\sigma(\lambda(x, y)))$ does not belong to $M(z)$.

Proof. If the atom $a$ is the label $\lambda(x, y)$ for the cover relation $x \prec y$, then the element $\max (\sigma(a))$ is not a smallest element in any block of the element $y$. That is, $\max (\sigma(a)) \notin$ $M(y)$. Since $M(z) \subseteq M(y)$, the result follows.

Proposition 4.6. The list $\left(a_{1}, \ldots, a_{n}\right)$ belongs to $M_{n, k}$ if and only if for all indices $p$, $1 \leq p \leq n$, the value $\max \left(\sigma\left(a_{p}\right)\right)$ does not appear among the support of the elements $a_{p+1}, \ldots, a_{n}$. That is, $\max \left(\sigma\left(a_{p}\right)\right) \notin \sigma\left(a_{p+1}\right) \cup \cdots \cup \sigma\left(a_{n}\right)$.

Proof. Let $c=\left\{\hat{0}=x_{0}<x_{1}<\cdots<x_{n}=\hat{1}\right\}$ be a maximal chain in the lattice $L_{n, k}$ so that, for $p<q, a_{p}=\lambda\left(x_{p-1}, x_{p}\right)$ and $a_{q}=\lambda\left(x_{q-1}, x_{q}\right)$. Since $x_{p} \leq x_{q-1}$, by Lemma 4.5 we have $\max \left(\sigma\left(a_{p}\right)\right) \notin M\left(x_{q-1}\right)$. However, by Corollary 4.4 we know $\sigma\left(a_{q}\right) \subseteq M\left(x_{q-1}\right)$. Hence we have $\max \left(\sigma\left(a_{p}\right)\right) \notin \sigma\left(a_{q}\right)$, so the labels of the chain $c$ satisfy the condition of the lemma.

Let $\left(a_{1}, \ldots, a_{n}\right)$ be a list of atoms which satisfies the condition in the lemma. For $0 \leq p \leq n$, let $x_{p}=a_{1} \vee \cdots \vee a_{p}$. We know that $\hat{0}=x_{0} \leq x_{1} \leq \cdots \leq x_{n}$ is a weakly increasing chain in $L_{n, k}$. We would like to prove that it is a maximal chain.

Since the entries $\max \left(\sigma\left(a_{1}\right)\right), \ldots, \max \left(\sigma\left(a_{n}\right)\right)$ are all distinct, we have that $M\left(x_{p-1}\right)$ is the disjoint union of $M\left(x_{p}\right)$ and $\left\{\max \left(\sigma\left(a_{p}\right)\right)\right\}$. Since $M\left(x_{0}\right)=\{1, \ldots, n\}$, we obtain $\left|M\left(x_{p}\right)\right|=n-p$, so the element $x_{p}$ has rank $p$. Hence the chain $\hat{0}=x_{0}<x_{1}<\cdots<x_{n}$ is a maximal chain in $L_{n, k}$. The elements in $\sigma\left(a_{p}\right)$ lie in $M\left(x_{p-1}\right)$, so they are smallest elements in their respective blocks. Hence the edge $\left(x_{p-1}, x_{p}\right)$ is labeled by the atom $a_{p}$. So we have that the list $\left(a_{1}, \ldots, a_{n}\right)$ belongs to the set $M_{n, k}$.

In order to state our main result, we need the notion of a weighted derivation. Here $\mathbb{P}$ denotes the positive integers.

Definition 4.7. Let $R$ be a graded algebra. A right weighted derivation $D$ is a function from $R \times \mathbb{P}$ to $R$ such that

$$
\begin{aligned}
D(\alpha \cdot u+\beta \cdot v, p) & =\alpha \cdot D(u, p)+\beta \cdot D(v, p), \\
D(1, p) & =0, \\
D(u \cdot v, p) & =D(u,|v|+p) \cdot v+u \cdot D(v, p),
\end{aligned}
$$

where $\alpha$ and $\beta$ are scalars and $|u|$ denotes the degree of the element $u$.

Let $K(v, p)$ be the right weighted derivation on $\mathbb{Z}\langle\mathbf{a}, \mathbf{b}\rangle$ such that $K(\mathbf{a}, p)=$ $K(\mathbf{b}, p)=(1+k \cdot p) \cdot \mathbf{a b}$. By induction on the degree of $v$, we may show the following result. 
Lemma 4.8. Let $v=v_{1} \cdots v_{n-1}$ be an $\mathbf{a b - m o n o m i a l . ~ T h e n ~} K(v, 1)$ is given by the sum

$$
K(v, 1)=\sum_{m=1}^{n-1}(1+k \cdot(n-m)) \cdot v_{1} \cdots v_{m-1} \cdot \mathbf{a b} \cdot v_{m+1} \cdots v_{n-1} .
$$

Using the weighted derivation $K$ we now obtain an expression for the ab-index of the Dowling lattice.

Theorem 4.9. The ab-index of the Dowling lattice $L_{n, k}$ satisfies the following recursion:

$$
\Psi\left(L_{n+1, k}\right)=\Psi\left(L_{n, k}\right) \cdot \mathbf{a}+K\left(\Psi\left(L_{n, k}\right), 1\right)+(1+k \cdot n) \cdot \mathbf{b} \cdot \Psi\left(L_{n, k}\right) .
$$

Proof. Consider a list $\lambda$ in the set $M_{n+1, k}$. There will be exactly one entry $\lambda_{m}$ in the list $\lambda=\left(\lambda_{1}, \ldots, \lambda_{n+1}\right)$ so that $\max \left(\sigma\left(\lambda_{i}\right)\right)=n+1$. Now observe that the list $\left(\lambda_{1}, \ldots, \lambda_{m-1}, \lambda_{m+1}, \ldots, \lambda_{n+1}\right)$ belongs to $M_{n, k}$.

Let $\lambda=\left(\lambda_{1}, \ldots, \lambda_{n}\right)$ be a list in $M_{n, k}$. Assume the ab-monomial $u(\lambda)$ is equal to $u_{1} \cdots u_{n-1}$. Let $m$ be an integer so that $0 \leq m \leq n$. We determine how many elements $a$ can be inserted in the $m$ th position of $\lambda$ so that we obtain a list in $M_{n+1, k}$. By Proposition 4.6 we have $\max (\sigma(a))$ must be $n+1$. Moreover, by Proposition 4.6 we have that $\max \left(\sigma\left(\lambda_{j}\right)\right)$ does not belong to $\sigma(a)$ for $1 \leq j \leq m$. These are the only conditions on $a$. Hence either $a$ is the atom labeled $n+1$ or it is of the form $(i, n+1, h)$, where $h$ can be chosen in $k$ possible ways and $i$ can be chosen in $n-m$ possible ways. Thus $a$ can be chosen in $1+k \cdot(n-m)$ possible ways.

The ab-monomial for the new list $\left(\lambda_{1}, \ldots, \lambda_{m-1}, a, \lambda_{m+1}, \ldots, \lambda_{n}\right)$ is $u_{1} \cdots u_{m-1}$. $\mathbf{a b} \cdot u_{m+1} \cdots u_{n-1}$ if $0<m<n$. If $m=0$ the ab-monomial is $\mathbf{b} \cdot u_{1} \cdots u_{n-1}=\mathbf{b} \cdot u$, while if $m=n$ the ab-monomial is $u_{1} \cdots u_{n-1} \cdot \mathbf{a}=u \cdot \mathbf{a}$. Summing over all positions $m$ we obtain

$$
\begin{array}{r}
u(\lambda) \cdot \mathbf{a}+\sum_{m=1}^{n-1}(1+k \cdot(n-m)) \cdot u_{1} \cdots u_{m-1} \cdot \mathbf{a b} \\
\cdot u_{m+1} \cdots u_{n-1}+(1+k \cdot n) \cdot \mathbf{b} \cdot u(\lambda) .
\end{array}
$$

By Lemma 4.8 the summation can be expressed in terms of the weighted derivation $K$. Hence we obtain the expression

$$
u(\lambda) \cdot \mathbf{a}+K(u(\lambda), 1)+(1+k \cdot n) \cdot \mathbf{b} \cdot u(\lambda)
$$

Summing over all $\lambda$ in $M_{n, k}$ we obtain the desired recursion for $\Psi\left(L_{n, k}\right)$.

Recall the characteristic polynomial of a graded poset $P$ is defined as $\chi(P)=$ $\sum_{\hat{0} \leq x \leq \hat{1}} \mu(\hat{0}, x) \cdot q^{\rho(x, \hat{1})}$. The characteristic polynomial of the Dowling lattice was first obtained in Proposition 7 of [11]. 
Corollary 4.10 [11]. The characteristic polynomial of the Dowling lattice $L_{n, k}$ is

$$
\chi\left(L_{n, k}\right)=\prod_{i=0}^{n-1}(q-1-k \cdot i) .
$$

Proof. Let $\bar{E}$ be the linear map from $\mathbb{Z}\langle\mathbf{a}, \mathbf{b}\rangle$ to $\mathbb{Z}[q]$ defined by

$$
\bar{E}(v)= \begin{cases}(-1)^{m} \cdot q^{k} & \text { if } v=\mathbf{b}^{m} \mathbf{a}^{k} \text { for some } m, k \geq 0, \\ 0 & \text { otherwise. }\end{cases}
$$

Then the characteristic polynomial is given by $\chi(P)=(q-1) \cdot \bar{E}(\Psi(P))$; see Proposition 5.3 of [7].

Apply the linear map $\bar{E}$ to Theorem 4.9. Observe that $\bar{E}(K(v, 1))=0$ since all the terms in $K(v, 1)$ contain the monomial ab. Hence we obtain

$$
\begin{aligned}
\bar{E}\left(\Psi\left(L_{n+1, k}\right)\right) & =\bar{E}\left(\Psi\left(L_{n, k}\right) \cdot \mathbf{a}\right)+(1+k \cdot n) \cdot \bar{E}\left(\mathbf{b} \cdot \Psi\left(L_{n, k}\right)\right) \\
& =(q-1-k \cdot n) \cdot \bar{E}\left(\Psi\left(L_{n, k}\right)\right) .
\end{aligned}
$$

Multiplying this identity with $q-1$, we have the recursion $\chi\left(L_{n+1, k}\right)=(q-1-k$. $n) \cdot \chi\left(L_{n, k}\right)$. Observing $\chi\left(L_{1, k}\right)=q-1$, we obtain the result.

By setting $q$ equal to zero in Corollary 4.10 we get Corollary 1 in [11].

Corollary 4.11 [11]. The Möbius function of the Dowling lattice $L_{n, k}$ is given by

$$
\mu\left(L_{n, k}\right)=(-1)^{n} \cdot \prod_{i=0}^{n-1}(1+k \cdot i)
$$

Since the Dowling lattice is $E L$-shellable, its chain complex is shellable. Hence we obtain:

Corollary 4.12. The chain complex of the Dowling lattice $L_{n, k}$ is homotopy equivalent to a wedge of spheres and only the highest homology is nontrivial. The dimension of the highest homology is given by $\prod_{i=0}^{n-1}(1+k \cdot i)$.

\section{The Braid Arrangements $A_{n}$ and $B_{n}$}

We now restrict our attention to the braid arrangements $A_{n}$ and $B_{n}$. These arrangements correspond to the cases $k=1$ and $k=2$ in $\mathcal{H}_{n, k}$, that is, when the arrangement $\mathcal{H}_{n, k}$ can be considered as a real arrangement.

The arrangement $A_{n}$ is most often described by the hyperplanes $x_{i}=x_{j}$ for $1 \leq i<$ $j \leq n+1$ in $(n+1)$-dimensional Euclidean space. This arrangement is not essential since each hyperplane contains the line $x_{1}=\cdots=x_{n+1}$. We obtain an essential hyperplane arrangement by setting the last variable $x_{n+1}$ equal to zero, yielding the arrangement corresponding to $\mathcal{H}_{n, 1}$. 
For $k$ equal to 1 and 2 let $R_{n, k}$ be the lattice of regions of the arrangement $\mathcal{H}_{n, k}$. Moreover, let $R_{n, 0}$ denote the lattice of regions of the coordinate hyperplanes, that is, $R_{n, 0}$ is the face lattice of the $n$-dimensional crosspolytope. By Theorem 3.4 the lattices $R_{n, 0}, R_{n, 1}$, and $R_{n, 2}$ have a c-2d-index. Using Theorem 4.9 we give an explicit recursion for the $\mathbf{c}-2 \mathbf{d}$-index of these three lattices.

Recall the definition of a right weighted derivation given in Definition 4.7. We have a similar notion of a left weighted derivation.

Definition 5.1. $\quad$ Let $R$ be a graded algebra. A left weighted derivation $D$ is a function from $R \times \mathbb{P}$ to $R$ such that $D$ satisfies (4.1) and (4.2), and

$$
D(u \cdot v, p)=D(u, p) \cdot v+u \cdot D(v,|u|+p) .
$$

We consider the weighted derivation $W$ on $\mathbb{Z}\langle\mathbf{c}, 2 \mathbf{d}\rangle$ that is defined by

$$
\begin{aligned}
W(\mathbf{c}, p) & =(1+k p) \cdot 2 \mathbf{d}, \\
W(2 \mathbf{d}, p) & =(1+k p) \cdot 2 \mathbf{d} \cdot \mathbf{c}+(1+k(p+1)) \cdot \mathbf{c} \cdot 2 \mathbf{d} .
\end{aligned}
$$

In our notation we suppress the fact that $W$ depends on the integer $k$. Observe that when $k=0$ the weighted derivation $W$ reduces to a derivation.

Theorem 5.2. The c-2d-index of $R_{n, k}, k=0,1,2$, satisfies the following recursion:

$$
\Psi\left(R_{n+1, k}\right)=\mathbf{c} \cdot \Psi\left(R_{n, k}\right)+W\left(\Psi\left(R_{n, k}\right), 1\right) .
$$

The case when $k=0$ was obtained in [16]. Hence it is enough to prove this theorem for $k=1$ and $k=2$.

The two weighted derivations $W$ and $K$ are related by the following identity.

Lemma 5.3. For any element $v$ in $\mathbb{Z}\langle\mathbf{a}, \mathbf{b}\rangle$ and any positive integer $p$

$$
W\left(\omega(v)^{*}, p\right)=\omega(K(v, p))^{*} .
$$

Proof. Since both sides are linear in $v$, it is enough to prove the statement for abmonomials. The proof is by induction on the degree of $v$. There are four base cases which are easy to verify, namely $v=1, v=\mathbf{a}, v=\mathbf{b}$, and $v=\mathbf{a b}$.

Consider now an ab-monomial $v$ different from the four base cases. We can write $v=u \cdot u^{\prime}$ such that $u, u^{\prime} \neq 1$ and $u$ does not end with a or $u^{\prime}$ does not begin with $\mathbf{b}$. That is, $u$ and $u^{\prime}$ have degrees smaller than $|v|$ and we have $\omega(v)=\omega(u) \cdot \omega\left(u^{\prime}\right)$. Also note that if $u$ ends with a, then all monomials in $K\left(u^{\prime}, p\right)$ will begin with a. Hence we know that $\omega\left(u \cdot K\left(u^{\prime}, p\right)\right)=\omega(u) \cdot \omega\left(K\left(u^{\prime}, p\right)\right)$. Similarly, if $u^{\prime}$ begins with $\mathbf{b}$, then all monomials in $K(u, p)$ will end with $\mathbf{b}$. That is, $\omega\left(K(u, p) \cdot u^{\prime}\right)=\omega(K(u, p)) \cdot \omega\left(u^{\prime}\right)$. 
Now we have

$$
\begin{aligned}
W\left(\omega\left(u \cdot u^{\prime}\right)^{*}, p\right) & =W\left(\omega\left(u^{\prime}\right)^{*} \cdot \omega(u)^{*}, p\right) \\
& =W\left(\omega\left(u^{\prime}\right)^{*}, p\right) \cdot \omega(u)^{*}+\omega\left(u^{\prime}\right)^{*} \cdot W\left(\omega(u)^{*},\left|u^{\prime}\right|+p\right) \\
& =\omega\left(K\left(u^{\prime}, p\right)\right)^{*} \cdot \omega(u)^{*}+\omega\left(u^{\prime}\right)^{*} \cdot \omega\left(K\left(u,\left|u^{\prime}\right|+p\right)\right)^{*} \\
& =\left[\omega(u) \cdot \omega\left(K\left(u^{\prime}, p\right)\right)+\omega\left(K\left(u,\left|u^{\prime}\right|+p\right)\right) \cdot \omega\left(u^{\prime}\right)\right]^{*} \\
& =\omega\left(u \cdot K\left(u^{\prime}, p\right)+K\left(u,\left|u^{\prime}\right|+p\right) \cdot u^{\prime}\right)^{*} \\
& =\omega\left(K\left(u \cdot u^{\prime}, p\right)\right)^{*} .
\end{aligned}
$$

This completes the induction.

We are now ready to give the proof of Theorem 5.2.

Proof of Theorem 5.2. By applying the map $v \longmapsto \omega(\mathbf{a} \cdot v)^{*}$ to Theorem 4.9 we obtain

$$
\begin{aligned}
\Psi\left(R_{n+1, k}\right) & =\omega\left(\mathbf{a} \cdot \Psi\left(L_{n+1, k}\right)\right)^{*} \\
& =\omega\left(\mathbf{a} \cdot \Psi\left(L_{n, k}\right) \cdot \mathbf{a}\right)^{*}+\omega\left(\mathbf{a} \cdot K\left(\Psi\left(L_{n, k}\right), 1\right)+(1+k \cdot n) \cdot \mathbf{a b} \cdot \Psi\left(L_{n, k}\right)\right)^{*} \\
& =\mathbf{c} \cdot \omega\left(\mathbf{a} \cdot \Psi\left(L_{n, k}\right)\right)^{*}+\omega\left(K\left(\mathbf{a} \cdot \Psi\left(L_{n, k}\right), 1\right)\right)^{*} \\
& =\mathbf{c} \cdot \omega\left(\mathbf{a} \cdot \Psi\left(L_{n, k}\right)\right)^{*}+W\left(\omega\left(\mathbf{a} \cdot \Psi\left(L_{n, k}\right)\right)^{*}, 1\right) \\
& =\mathbf{c} \cdot \Psi\left(R_{n, k}\right)+W\left(\Psi\left(R_{n, k}\right), 1\right),
\end{aligned}
$$

where the fourth step is by Lemma 5.3.

It is now straightforward to compute the following table:

\begin{tabular}{c|l|l}
$n$ & \multicolumn{1}{|c|}{$\Psi\left(R_{n, 1}\right)$} & \multicolumn{1}{c}{$\Psi\left(R_{n, 2}\right)$} \\
\hline 0 & 1 & 1 \\
1 & $\mathbf{c}$ & $\mathbf{c}$ \\
2 & $\mathbf{c}^{2}+2 \cdot 2 \mathbf{d}$ & $\mathbf{c}^{2}+3 \cdot 2 \mathbf{d}$ \\
3 & $\mathbf{c}^{3}+11 \cdot \mathbf{c} \cdot 2 \mathbf{d}+6 \cdot 2 \mathbf{d} \cdot \mathbf{c}$ & $\mathbf{c}^{3}+23 \cdot \mathbf{c} \cdot 2 \mathbf{d}+12 \cdot 2 \mathbf{d} \cdot \mathbf{c}$ \\
4 & $\mathbf{c}^{4}+59 \cdot \mathbf{c}^{2} \cdot 2 \mathbf{d}+60 \cdot \mathbf{c} \cdot 2 \mathbf{d} \cdot \mathbf{c}$ & $\mathbf{c}^{4}+191 \cdot \mathbf{c}^{2} \cdot 2 \mathbf{d}+186 \cdot \mathbf{c} \cdot 2 \mathbf{d} \cdot \mathbf{c}$ \\
& $\begin{array}{r}+14 \cdot 2 \mathbf{d} \cdot \mathbf{c}^{2}+46 \cdot(2 \mathbf{d})^{2} \\
\end{array}$ & $+36 \cdot 2 \mathbf{d} \cdot \mathbf{c}^{2}+146 \cdot(2 \mathbf{d})^{2}$
\end{tabular}

For instance, to compute $\Psi\left(R_{3,1}\right)$ we have

$$
\begin{aligned}
\Psi\left(R_{3,1}\right) & =\mathbf{c} \cdot \Psi\left(R_{2,1}\right)+W\left(\Psi\left(R_{2,1}\right), 1\right) \\
& =\mathbf{c} \cdot\left(\mathbf{c}^{2}+2 \cdot 2 \mathbf{d}\right)+W\left(\mathbf{c}^{2}+2 \cdot 2 \mathbf{d}, 1\right) \\
& =\mathbf{c}^{3}+2 \cdot \mathbf{c} \cdot 2 \mathbf{d}+W(\mathbf{c}, 1) \cdot \mathbf{c}+\mathbf{c} \cdot W(\mathbf{c}, 2)+2 \cdot W(2 \mathbf{d}, 1) \\
& =\mathbf{c}^{3}+2 \cdot \mathbf{c} \cdot 2 \mathbf{d}+2 \cdot 2 \mathbf{d} \cdot \mathbf{c}+3 \cdot \mathbf{c} \cdot 2 \mathbf{d}+4 \cdot 2 \mathbf{d} \cdot \mathbf{c}+6 \cdot \mathbf{c} \cdot 2 \mathbf{d} \\
& =\mathbf{c}^{3}+11 \cdot \mathbf{c} \cdot 2 \mathbf{d}+6 \cdot 2 \mathbf{d} \cdot \mathbf{c} .
\end{aligned}
$$

\section{Concluding Remarks}

The permutahedron $P_{n}$ is the $n$-dimensional polytope whose vertices are the $(n+1)$ ! permutations in the symmetric group on $n+1$ elements. In other words, $P_{n}$ lies in the 
hyperplane $x_{1}+\cdots+x_{n+1}=\left(\begin{array}{c}n+2 \\ 2\end{array}\right)$ and has vertices $(\pi(1), \ldots, \pi(n+1))$, where $\pi$ ranges over all permutations on $n+1$ elements. Similarly, let the signed permutahedron $P_{n}^{ \pm}$be the convex hull of the points $( \pm \pi(1), \ldots, \pm \pi(n))$, where $\pi$ ranges over all permutations on $n$ elements. Examples of these polytopes include the line segments $P_{1}$ and $P_{1}^{ \pm}$, the hexagon $P_{2}$, the octagon $P_{2}^{ \pm}$, the truncated octahedron $P_{3}$, and the rhombitruncated cuboctahedron $P_{3}^{ \pm}$.

It is well known that the face lattice of the permutahedron $\mathcal{L}\left(P_{n}\right)$ is given by the dual poset of $R_{n, 1}$. Moreover, the lattice $R_{n, 1}$ has a combinatorial interpretation as the ordered partition lattice; see for instance [8]. Similarly, the face lattice of the signed permutahedron $\mathcal{L}\left(P_{n}^{ \pm}\right)$is the dual poset of $R_{n, 2}$. We present the following combinatorial description of the lattice $R_{n, 2}$.

A signed block $\widetilde{B}$ is a nonempty set $B$ with a function $f: B \rightarrow\{-1,1\}$. This notion differs from that of an enriched block which was defined in Section 4 since we now consider the two signed blocks $(B, f)$ and $(B,-f)$ to be different. An ordered signed partition $\tilde{\pi}$ is an ordered partition $\pi=\left(B_{1}, \ldots, B_{m}\right)$, where each block $B_{i}$ is signed by a function $f_{i}$. If $\widetilde{B}$ and $\widetilde{C}$ are two signed blocks, then we may consider their union as a signed block. Define the lattice $R_{n, 2}^{\prime}$ to be the set

$$
\begin{aligned}
R_{n, 2}^{\prime}= & \{(\tilde{\pi}, Z): Z \subseteq\{1, \ldots, n\} \\
& \text { and } \tilde{\pi} \text { is an ordered signed partition of } \bar{Z}=\{1, \ldots, n\}-Z\} .
\end{aligned}
$$

The order relation on $R_{n, 2}^{\prime}$ is given by

$$
\begin{aligned}
\left(Z,\left(\widetilde{B}_{1}, \widetilde{B}_{2}, \ldots, \widetilde{B}_{m}\right)\right) & <\left(Z \cup B_{1},\left(\widetilde{B}_{2}, \ldots, \widetilde{B}_{m}\right)\right), \\
\left(Z,\left(\widetilde{B}_{1}, \ldots, \widetilde{B}_{i}, \widetilde{B}_{i+1}, \ldots, \widetilde{B}_{m}\right)\right) & <\left(Z,\left(\widetilde{B}_{1}, \ldots, \widetilde{B}_{i} \cup \widetilde{B}_{i+1}, \ldots, \widetilde{B}_{m}\right)\right) .
\end{aligned}
$$

The first relation says that the first block is allowed to merge with the zero set. The second relation says that two adjacent blocks are allowed to be merged together.

Given $(Z, \tilde{\pi}) \in R_{n, 2}^{\prime}$, where the zero set contains the elements $j_{0,1}, \ldots, j_{0, m_{0}}$ and the $i$ th block contains the elements $j_{i, 1}, \ldots, j_{i, m_{i}}$, the corresponding region of $B_{n}$ is constructed by

$$
\begin{aligned}
0 & =x_{j_{0,1}}=\cdots=x_{j_{0, m_{0}}} \\
& <f_{1}\left(j_{1,1}\right) \cdot x_{j_{1,1}}=\cdots=f_{1}\left(j_{1, m_{1}}\right) \cdot x_{j_{1, m_{1}}} \\
& <\cdots \\
& <f_{m}\left(j_{m, 1}\right) \cdot x_{j_{m, 1}}=\cdots=f_{m}\left(j_{m, m_{m}}\right) \cdot x_{j_{m, m_{m}}} .
\end{aligned}
$$

For $k=0,1$, or 2 , the lattice of regions $R_{n, k}$ corresponds to a root system. The set of exponents of the root system is $1,1+k, \ldots, 1+k \cdot(n-1)$. These numbers appear as weights in the weighted derivations $W$ and $K$. This suggests that a similar recursion should hold for other root systems in general. Hence a good question to consider is what is the $\mathbf{c}$-2d-index of the lattice of regions for the simple sporadic root systems.

An interesting challenge is to find a recursion for the root system $D_{n}$. Recall that the corresponding hyperplane arrangement is

$$
x_{i}= \pm x_{j} \quad \text { for } \quad 1 \leq i<j \leq n,
$$


for $n \geq 4$. What can be said about the ab-index of the complex $D_{n}$ arrangement

$$
z_{i}=\zeta^{h} \cdot z_{j} \quad \text { for } \quad 1 \leq i<j \leq n \quad \text { and } \quad 0 \leq h \leq k-1 \text {, }
$$

where $\zeta$ is a $k$ th primitive root of unity?

The homology of the partition lattice $\Pi_{n+1}=L_{n, 1}$ has been extensively studied in order to find representations of the symmetric group; see [23] and [28]. In the same spirit, Wachs has studied the signed partition lattice $L_{n, 2}$; see [29]. What can be said about the representations of the symmetric group arising from the Dowling lattice $L_{n, k}$ ? A related question is to find an explicit basis for the highest homology group of the Dowling lattice, the dimension of which is given in Corollary 4.12 .

\section{Acknowledgments}

We thank Louis Billera for helpful discussions and the two referees for their comments and suggestions to improve the exposition.

\section{References}

1. M. Bayer, The extended $f$-vectors of 4-polytopes, J. Combin. Theory Ser. A 44 (1987), 141-151.

2. M. Bayer and L. Billera, Generalized Dehn-Sommerville relations for polytopes, spheres and Eulerian partially ordered sets, Invent. Math. 79 (1985), 143-157.

3. M. Bayer and A. Klapper, A new index for polytopes, Discrete Comput. Geom. 6 (1991), 33-47.

4. M. M. Bayer and C. W. Lee, Combinatorial aspects of convex polytopes, in Handbook of Convex Geometry, Vol. A (P. M. Gruber and J. M. Wills, eds.), Chapter 2.3, Elsevier, Amsterdam, 1993.

5. M. Bayer and B. Sturmfels, Lawrence polytopes, Canad. J. Math. 42 (1990), 62-79.

6. L. J. Billera, R. Ehrenborg, and M. Readdy, The cd-index of zonotopes and arrangements, in: Mathematical Essays in Honor of Gian-Carlo Rota (B. E. Sagan and R. P. Stanley, eds.), Birkhäuser Boston, Boston, to appear.

7. L. J. Billera, R. Ehrenborg, and M. Readdy, The c-2d-index of oriented matroids, J. Combin. Theory Ser. A 80 (1997), 79-105.

8. L. J. Billera and A. Sarangarajan, The combinatorics of permutation polytopes, in Algebraic Combinatorics (L. Billera, C. Greene, R. Simion, and R. Stanley, eds.), DIMACS Series in Discrete Mathematics and Theoretical Computer Science, American Mathematical Society, Providence, RI, 1996.

9. A. Björner, Shellable and Cohen-Macaulay partially ordered sets, Trans. Amer. Math. Soc. 260 (1980), 159-183.

10. A. Björner, M. Las Vergnas, B. Sturmfels, N. White, and G. Ziegler, Oriented Matroids, Cambridge University Press, Cambridge, 1993.

11. T. A. Dowling, A $q$-analog of the partition lattice, in A Survey of Combinatorial Theory (J. N. Srivastava et al., eds.), pp. 101-115, North-Holland, Amsterdam, 1973.

12. T. A. Dowling, A class of geometric lattices based on finite groups, J. Combin. Theory Ser. B 14 (1973), 61-86.

13. R. Ehrenborg, On posets and Hopf algebras, Adv. in Math. 119 (1996), 1-25.

14. R. Ehrenborg and G. Hetyei, Flags and shellings of Eulerian cubical posets, Preprint 1998.

15. R. Ehrenborg and M. Readdy, The r-cubical lattice and a generalization of the cd-index, European J. Combin. 17 (1996), 709-725.

16. R. Ehrenborg and M. Readdy, Coproducts and the cd-index, to appear in J. Algebraic Combin.

17. G. Hetyei, On the $c d$-variation polynomials of André and simsun permutations, Discrete Comput. Geom. 16 (1996), 259-275. 
18. T. Józefiak and B. Sagan, Basic derivations for subarrangements of Coxeter arrangements, J. Algebraic Combin. 2 (1993), 291-320.

19. G. Kalai, Rigidity and the lower bound theorem, I, Invent. Math. 88 (1987), 125-151.

20. P. Orlik and H. Terao, Arrangements of Hyperplanes, Grundlehren der matematischen Wissenschaften, Vol. 300, Springer-Verlag, New York, 1992.

21. M. Purtill, André permutations, lexicographic shellability and the $c d$-index of a convex polytope, Trans. Amer. Math. Soc. 338 (1993), 77-104.

22. R. P. Stanley, Finite lattices and Jordan-Hölder sets, Alg. Univ. 4 (1974), 361-371.

23. R. P. Stanley, Some aspects of groups acting on finite posets, J. Combin. Theory Ser. A 32 (1982), 132-161.

24. R. P. Stanley, Enumerative Combinatorics, Vol. I, Wadsworth and Brooks/Cole, Pacific Grove, CA, 1986.

25. R. P. Stanley, Flag $f$-vectors and the $c d$-index, Math. Z. 216 (1994), 483-499.

26. R. P. Stanley, A survey of Eulerian posets, in: Polytopes: Abstract, Convex, and Computational (T. Bisztriczky, P. McMullen, R. Schneider, and A. I. Weiss, eds.), NATO ASI Series C, Vol. 440, Kluwer, Dordrecht, 1994.

27. R. P. Stanley, Enumerative Combinatorics, Vol. II, Cambridge University Press, Cambridge, to appear.

28. S. Sundaram, The homology representations of the symmetric group on Cohen-Macaulay subposets of the partition lattice, Adv. in Math 104 (1994), 225-296.

29. M. Wachs, Geometrically constructed homology bases for real hyperplane intersection lattices, talk at Enumeration and Partially Ordered Sets Workshop, Mathematical Sciences Research Institute, October 14th, 1996.

30. T. Zaslavsky, Facing up to arrangements: face count formulas for partitions of space by hyperplanes, Mem. Amer. Math. Soc. 154 (1975), 1-102.

31. G. M. Ziegler, Lectures on Polytopes, Springer-Verlag, New York, 1995.

Received May 1, 1997, and in revised form August 4, 1997. 